\title{
Variability of Jovian ion winds: an upper limit for enhanced Joule heating
}

\author{
M. B. Lystrup ${ }^{1,2}$, S. Miller ${ }^{1,2}$, T. Stallard ${ }^{1}$, C. G. A. Smith ${ }^{1}$, and A. Aylward ${ }^{1}$ \\ ${ }^{1}$ Department of Physics \& Astronomy, University College London, Gower Street, London WC1E 6BT, UK \\ ${ }^{2}$ Visiting Astronomer at the Infrared Telescope Facility, which is operated by the University of Hawaii under Cooperative \\ Agreement no. NCC 5-538 with the National Aeronautics and Space Administration, Science Mission Directorate, Planetary \\ Astronomy Program
}

Received: 29 September 2006 - Revised: 19 March 2007 - Accepted: 27 March 2007 - Published: 8 May 2007

\begin{abstract}
It has been proposed that short-timescale fluctuations about the mean electric field can significantly increase the upper atmospheric energy inputs at Jupiter, which may help to explain the high observed thermospheric temperatures. We present data from the first attempt to detect such variations in the Jovian ionosphere. Line-of-sight ionospheric velocity profiles in the Southern Jovian auroral/polar region are shown, derived from the Doppler shifting of $\mathrm{H}_{3}^{+}$ infrared emission spectra. These data were recently obtained from the high-resolution CSHELL spectrometer at the NASA Infrared Telescope Facility. We find that there is no variability within this data set on timescales of the order of one minute and spatial scales of $640 \mathrm{~km}$, putting upper limits on the timescales of fluctuations that would be needed to enhance Joule heating.
\end{abstract}

Keywords. Magnetospheric physics (Auroral phenomena) Ionosphere (Ionosphere-atmosphere interactions; Planetary ionospheres)

\section{Introduction}

The origin of the remarkably high thermospheric temperatures of Jupiter (and indeed all the gas giant planets), confirmed by measurements from the Voyager (Atreya, 1986) and Galileo spacecraft (Seiff et al., 1997) and from groundbased observations (Stallard et al., 2002) to be $\sim 900 \mathrm{~K}$, remains unexplained. Solar heating cannot, by itself, account for these temperatures (Strobel and Smith, 1973) and various additional energy sources have been suggested. From as early as 1983, energy from particles precipitated into the upper atmosphere in auroral regions has been considered (Waite et al., 1983; Grodent and Gérard, 2001). Normal Joule heating (calculated using the average ionospheric electric field)

Correspondence to: M. B. Lystrup

(mlystrup@ucl.ac.uk)
(Cowley et al., 2005) and ion drag (Miller et al., 2000) have been suggested as possible heat sources, as well as gravity waves (Young et al., 1997) and acoustic waves (Schubert et al., 2003). However, none of these additional energy inputs have been demonstrated unambiguously to explain the energy gap (Matcheva and Strobel, 1999; Hickey et al., 2000).

Joule heating in the upper atmosphere is the result of relative motion between neutrals and ions - the thermalization of kinetic energy (Cowley et al., 2004). Electric fields are generated by plasma flows in the magnetosphere and map along magnetic field lines into the upper atmosphere, resulting in bulk ion flows. The jovian equatorial plasma sheet, constantly fed by material from Io, receives rotational energy from Jupiter that keeps the plasma sheet in corotation with the planet. At around 20 Jovian radii from the planet, corotation begins to break down (Hill, 1979), generating a current system that closes through the ionosphere. This mechanism implies an equatorward field across the auroral oval, where a near-perpendicular planetary magnetic field exists. The result is a westward (Hall) ion drift - an ion wind - around the oval, with a magnitude given by $v_{\text {ion }}=E_{e q} / B_{J}$, where $E_{e q}$ is the equatorward electric field and $B_{J}$ is the Jovian magnetic field at the auroral oval. These ion winds were first detected in the Jovian aurora by Rego et al. (1999) and subsequent observations have been used to probe the dynamics of and energy balance in the Jovian upper atmosphere (Stallard et al., 2001). Typically, $v_{\text {ion }}$ is of the order of $1 \mathrm{kms}^{-1}$; with $B_{J} \approx 10^{-3}$ Tesla, $E_{e q}$ must therefore have a value around $1 \mathrm{Vm}^{-1}$.

Intermittent variations in magnetospheric conditions over timescales of a few hours have been discussed by Cowley and Bunce (2001) and Southwood and Kivelson (2001). In particular, expansion of the jovian magnetosphere following solar wind compression was found to result in increased auroral activity and, presumably, accompanying heating effects. Although no studies on quite this timescale have been attempted, Melin et al. (2006) showed that changes in

Published by Copernicus GmbH on behalf of the European Geosciences Union. 


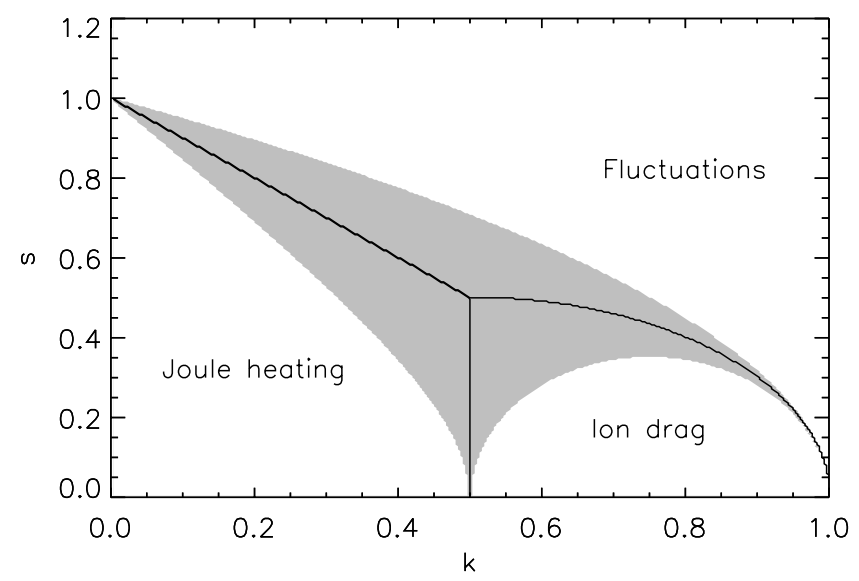

Fig. 1. The standard deviation of the fluctuations, $s$, is plotted versus $k$ showing the the relative importance of heating mechanisms. The unshaded region indicates where a heating source is dominant by greater than $50 \%$. Figure from Smith et al. (2005).

magnetospheric inputs to Jupiter's ionosphere over several Jovian rotations could result in considerable excess heating of the upper atmosphere. All of these studies, however, deal with variations whose timescales are on the order of, or considerably greater than, the time taken for the thermosphere (the main component of the upper atmosphere) to respond to changes in the velocity of the ions: for Jupiter, modelling by Millward et al. (2005) indicates that this thermospheric response time should be on the order of $1500 \mathrm{~s}$.

For the Earth, Codrescu et al. (1995) suggested that calculations considering only the average electric field, ignoring fluctuations on timescales shorter than the thermospheric response time, underestimate Joule heating for the highlatitude regions. The first direct evidence supporting this was found by northern auroral measurements of Earth's thermosphere by Aruliah et al. (2005). They found that the variations on timescales of one minute in the electric field do indeed contribute significantly to Joule heating. Smith et al. (2005) argued that, similar to the case in Earth's ionosphere, persistent short-timescale fluctuations about the mean electric field could significantly increase the average Joule heating in the upper atmosphere of Jupiter. To explain this, they expressed the Joule heating and the ion drag (the kinetic energy) in the rest frame of the neutrals as, respectively:

$Q_{J}=(1-k)^{2} \Sigma_{P} E^{2}$

$Q_{I D}=k(1-k)^{2} \Sigma_{P} E^{2}$

where $\Sigma_{P}$ is the Pedersen conductivity, $E$ is the electric field, and $k$ is defined as $k=v_{n} / v_{i}$, where $v_{n}$ is the neutral wind velocity and $v_{i}$ is the westward ion drift velocity. They then considered the full, fluctuating electric field as a sum of the mean electric field $E$ and the random fluctuations $f(t)$, which have some statistical distribution with a mean of zero and variance $\overline{f^{2}}=s^{2}$ :

$E_{f}(t)=E(1+f(t))$

The neutrals cannot respond to changes in the ion wind on short timescales and are therefore insensitive to rapid variations in the electric field. Thus, Smith et al. (2005) showed that the time-averaged Joule heating becomes:

$\overline{Q_{J f}}=\left[(1-k)^{2}+s^{2}\right] \Sigma_{P} E^{2}$

The magnitude of the Joule heating component from the fluctuating electric field, $s^{2} \Sigma_{P} E^{2}$, is independent of $k$ and depends only on the variance. However, the relative importance of this component from fluctuations depends also on $k$. The value of $k$ is unknown, but some models have predicted it to be as high as 0.7 for the peak of the Jovian ion density (Millward et al., 2005). Figure 1 shows the relative importance of Joule heating, Joule heating from electric field fluctuations, and ion drag (which is unaffected by electric field fluctuations) for a range of $k$ and standard deviation $(s)$ values. If $s$ is 1.0, which means the fluctuations in the field are of magnitude of the mean of the field, the contributed Joule heating is significant no matter the value of $k$. The fluctuations in Earth's electric field observed by Aruliah et al. (2005) have $s$ of 1.0. (Note that $k$ is not generally considered a useful parameter with respect to Earth.) For $k$ values predicted by models of Jupiter (Cowley and Bunce, 2001), around 0.5, the standard deviation of the fluctuations needed to enhance Joule heating significantly must be around 0.6.

These fluctuations do not have a preferential time scale, as long as the time scale is short enough that the neutrals do not have enough time to respond to the change in electric field on order of $1500 \mathrm{~s}$ (Millward et al., 2005); the ions respond immediately. Aruliah et al. (2005) found fluctuations significant on the order of one minute, but insignificant for Joule heating on the order of $15 \mathrm{~min}$. It is also unknown if a preferred size scale exists for the fluctuations.

Evidence for turbulence in the Jovian middle magnetosphere has been seen in an analysis of Galileo data and it has been suggested that this turbulence could affect resistivity along magnetic field lines and alter the Hill (1979) mechanism that couples the Jovian atmosphere with the magnetosphere (Saur et al., 2002). This turbulence might also drive electric field fluctuations that would lead to an enhanced Joule heating effect. Given a constant value of $B_{J}$, fluctuations in the ionospheric electric field, $E_{e q}$, would result in corresponding variations in $v_{\text {ion }}$. Our study is the first attempt to detect velocity fluctuations in the Jovian system to look for this augmented Joule heating. (Saur et al., 2002) found that fluctuations were typically present in all of the Galileo data they examined, and thus a persistent feature of the Jovian magnetosphere. Their analysis was sensitive to timescales between $24 \mathrm{~s}$ and $4500 \mathrm{~s}$ at a distance of $26 R_{J}$ 
from the planet, at which distance they estimated Alfvén travel times in the magnetosphere to be $375 \mathrm{~s}$ per Jovian radius $\left(1 R_{J}=71492 \mathrm{~km}\right)$. This would make the Alfvén travel time from $20 R_{J}$ to the planet itself be $7500 \mathrm{~s}$.

\section{Observations}

On 5 May 2006 the CSHELL echelle spectrometer (Greene et al., 1993) at the NASA Infrared Telescope Facility (IRTF) on Mauna Kea, Hawaii was used to collect these data. We chose to observe the $\mathrm{Q}\left(1,0^{-}\right)$emission line in the $\nu_{2}$ fundamental vibrational band of the $\mathrm{H}_{3}^{+}$molecular ion. This emission line has been used successfully to probe Jupiter's upper atmosphere in many other studies (Rego et al., 1999; Stallard et al., 2001, 2004; Melin et al., 2005). Ground-based infrared observations of the $\mathrm{Q}\left(1,0^{-}\right)$line and other bright emission features of $\mathrm{H}_{3}^{+}$are possible because they lie within Earth's atmospheric L' window and fall within a wavelength region in which Jupiter's IR continuum spectrum is suppressed by stratospheric methane absorption.

CSHELL's circular variable filter was set to pick out $3.953 \mu \mathrm{m}$, the wavelength of the $\mathrm{Q}\left(1,0^{-}\right)$line. The instrument was rotated at an angle of $71.8^{\circ}$ such that the slit was positioned to cut east-west across the entire auroral oval, perpendicular to Jupiter's rotational axis which had a position angle of $18.2^{\circ}$ on 5 May (see Fig. 2). We attempted to keep the slit in the same position throughout the night, but some drift resulted from the limitations of the guiding system's ability to track an extended object. Images of Jupiter at Kband in CSHELL's direct imaging mode were made and used to align the slit along the polar limb and calculate telescope offsets. Images at $3.953 \mu \mathrm{m}$ were used at the data analysis stage to find the exact slit position on the planet.

Apparent wavelength shifts can be introduced due to uneven illumination across a slit, and the surface brightness of the Jovian aurora varies on a variety of spatial scales. In previous studies, CSHELL images taken at $3.953 \mu \mathrm{m}$ have been used to correct for the minor discrepancies due to this effect (Stallard et al., 2001). In recent years, however, the quality of these images has degraded such that corrections for spatial effects are difficult to apply. In order to minimize this effect, the narrowest slit width available on CSHELL, 0.5", was chosen giving spatial resolution of 0.2 " per pixel. At $3.953 \mu \mathrm{m}$, this slit gives a resolving power of $\lambda / \Delta \lambda \sim 40000$.

We made spectral observations of Jupiter in an ABBA nod-to-sky pattern (where $\mathrm{A}$ is an object exposure and B is a sky exposure), but because we were interested in minute-to-minute variations, we also made observations in an $\mathrm{AB} / \mathrm{A} / \mathrm{AB}$ pattern to minimize the time between sky exposures. For Jovian spectra we used an integration time of $50 \mathrm{~s}$, as that time has previously yielded good signal-to-noise. After 10 Jupiter exposures, we took an image at K-band and at $3.953 \mu \mathrm{m}$. This observing strategy was implemented on both the Northern and the Southern aurorae; the Northern aurora

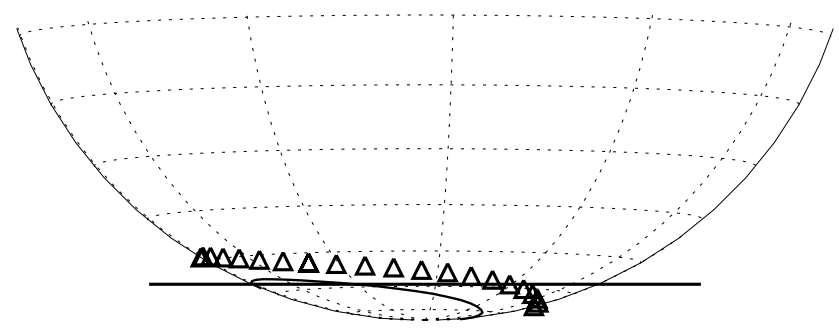

Fig. 2. A schematic diagram of the slit position across the southern auroral region of Jupiter for a CML of 334. The solid curve is the $30 R_{J}$ oval and the triangles are the Io footprint, both from the VIP4 model of Connerney et al. (1998).

was relatively weak, however, so only the higher-intensity Southern data are presented for this analysis. The relative weakness of the northern auroral emission is an interesting observation, which we have noted in other (unpublished) data, and which we shall address in a future publication.

\section{Data reduction and analysis}

Spectra were reduced using standard infrared techniques of sky-subtracting, flat-fielding, removing bad pixels, and wavelength calibration. For each spectral row across the planet, the spectrum was fitted with a Gaussian fitting procedure. Peak intensities, line positions, and line widths for the spectral rows were recorded.

Velocity profiles were calculated from the Dopplershifting of the $\mathrm{Q}\left(1,0^{-}\right)$line. In addition to the actual velocity of the ion winds, this measured velocity includes components due to the rotation of the planet, the nonlinearity of the array's wavelength scale, and an arbitrary shift from zero. The details of correcting for these factors can be found in Stallard et al. (2001). The zero-position of the velocity was set where the emission from the $\mathrm{H}_{3}^{+}$has zero velocity - on the dusk side of the planet between the auroral oval and the limb. $\mathrm{H}_{3}^{+}$emission does exist in this region, but it is not associated with the electrojet or other ion wind systems. The velocity profiles presented here have, as explained above, not been subject to correction for uneven illumination across the slit. This may affect the actual velocity measurements, although it is minimized in the Southern Hemisphere where the viewing angle of the oval from Earth tends to average out these effects. After these corrections we are left with the line-of-sight, non-spatially-corrected velocity profiles (Stallard et al., 2001):

$v_{\text {line-of-sight }}=v_{\text {measured }}+v_{\text {spatial }}$

The morphology of the infrared auroral oval is relatively stable on short timescales and we used the intensity profiles as a check of the positioning of the slit on the planet. Series 

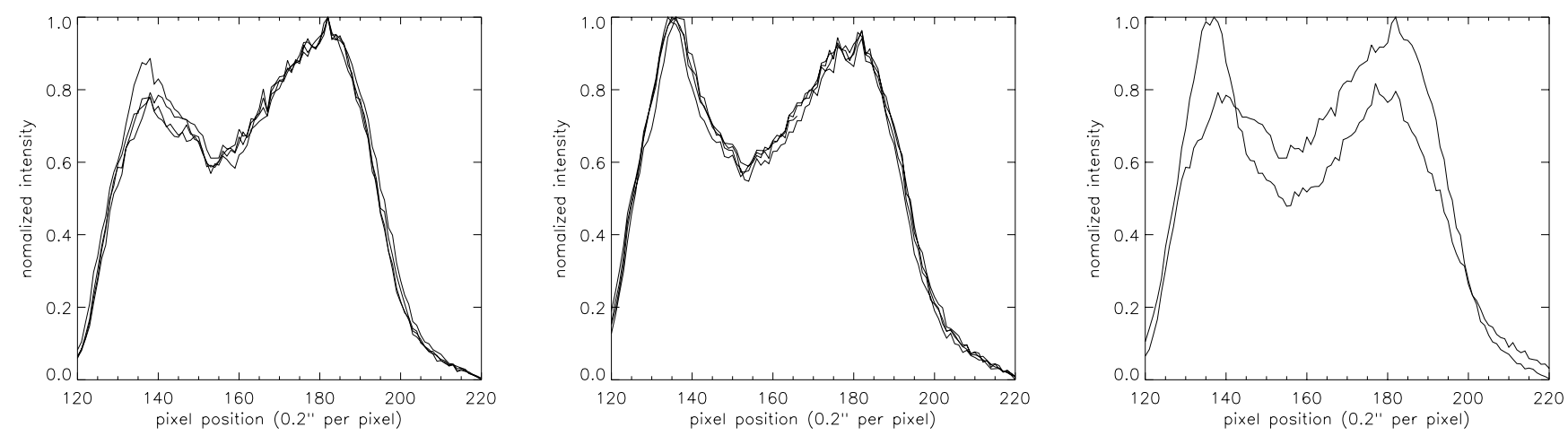

Fig. 3. The first and second plot shows the intensity profiles of spectra in Group 1 and Group 2, respectively. The last plot shows two consecutive spectra, taken only $55 \mathrm{~s}$ apart, that have clearly different intensity morphologies, and were thus taken at different latitudes due to an inability to keep the slit stationary on the planet.

Table 1. Spectra considered in this study.

Group 1

Group 2

\begin{tabular}{cccccccc}
\hline File No. & UT & CML & Airmass & File No. & UT & CML & Airmass \\
\hline 151 & $10: 36: 04.05$ & 330.244 & 1.221 & 159 & $10: 43: 26.09$ & 334.701 & 1.225 \\
156 & $10: 40: 42.00$ & 333.047 & 1.223 & 160 & $10: 44: 21.06$ & 335.255 & 1.226 \\
157 & $10: 41: 37.00$ & 333.601 & 1.224 & 161 & $10: 45: 16.06$ & 335.810 & 1.227 \\
158 & $10: 42: 31.09$ & 334.146 & 1.225 & 162 & $10: 46: 11.06$ & 336.365 & 1.227 \\
\hline
\end{tabular}

of spectra with the same intensity profiles were assumed to have been taken to be at the same latitude. These conditions were satisfied for two sets of four spectra, as listed in Table 1 . In both groups the intensity profiles closely match, as seen in Fig. 3. Consecutive spectra did not always fit this condition; the same figure shows another pair of consecutive spectra with very different intensity profiles, clearly affected by drift in the telescope pointing, which were not analyzed further.

For each group, the mean of the four velocity profiles and each profile's difference from that mean were calculated. For comparison, model profiles were created assuming a normal distribution of fluctuations with different variances and with mean equal to the mean of the observed velocity profiles. The model profiles were smoothed to 3 pixels to simulate the 0.6 " seeing conditions that existed during our observations. These simple model profiles show what we might expect our observed velocity profiles to look like if fluctuations exist.

\section{Results and discussion}

For both groups of spectra showing near-identical intensity profiles, the mean of the four velocity profiles and each profile's difference from that mean were calculated. These are shown for Group 1 in Fig. 4. For comparison, model profiles were created assuming a normal distribution of fluctuations with different variances and with mean equal to the mean of the observed velocity profiles. The model profiles were smoothed to 3 pixels to simulate the 0.6 " seeing conditions that existed during our observations. These simple model profiles reveal what we might expect our observed velocity profiles to look like if fluctuations do exist.

Figure 2 shows that the CSHELL slit crosses the main auroral oval, which lies a little equatorward of the $30 R_{J}$ oval, on the dawn limb of the planet at the ansa. That means that we view the auroral electrojet more or less parallel to the flow of the ions around the oval. This shows up as a redshift of approximately $-1 \mathrm{kms}^{-1}$ around pixels $135-138$. Further west (on the planet) the northern edge of the slit is more fully illuminated than the southern, as a result of the curvature of the planet. The effect of this uneven illumination is to produce an additional apparent redshift, such that the velocity appears to be $-2 \mathrm{kms}^{-1}$ at pixels $122-123$. Figure 2 also shows that the slit cuts across the duskward side of the oval at a point where the ion flow around the oval is almost perpendicular to our line-of-sight. This results in only a barely detectable blue shift of $<+100 \mathrm{~ms}^{-1}$ around pixels $180-185$, before the velocity drops to zero on the body of the planet towards the dusk limb. 

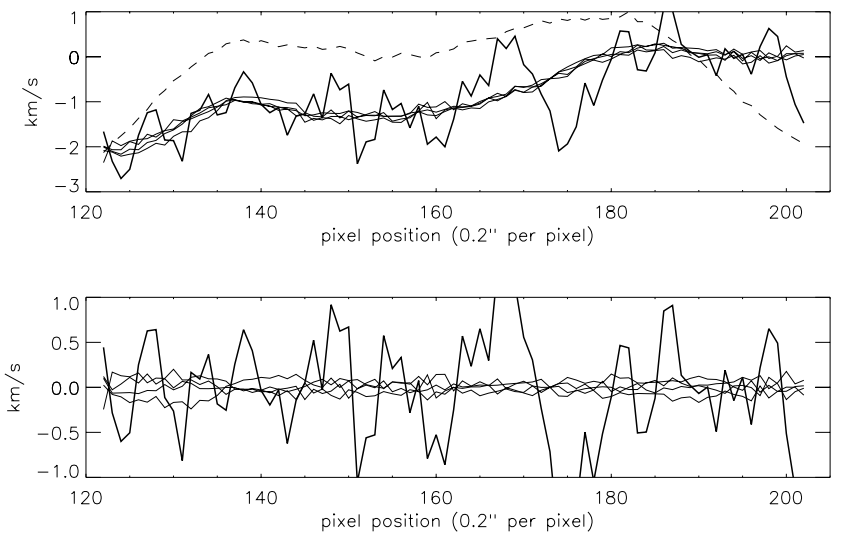

Fig. 4. The four spectra from Group 1 are shown (top) with variations from the group average (bottom). The bold lines are the model profile and fluctuations from the Group 1 average assuming $s=1.0$, smoothed to three pixels to estimate seeing effects. The large negative velocities seen between pixels $\sim 122$ and 132 are due to uneven illumination across the slit as it crosses the dawn limb of the planet. The dashed curve is the average intensity profile for this group of spectra, scaled to the velocity.

In bold in Fig. 4 is a model profile with a mean equal to the Group 1 mean and standard deviation $s=1$. For this model the velocity structure is dominated by $1 \mathrm{kms}^{-1}$ variations. But the observed variations are of less than $200 \mathrm{~ms}^{-1}$, which is at the resolution limit of CSHELL. Figure 5 shows the corresponding data for Group 2, this time plotted with the model profile using $s=0.5$, the lowest estimate that significantly contributes to heating (Smith et al., 2005). The model fluctuations also remain significantly larger than those we observed. We therefore conclude that we are not able to observe fluctuations that would contribute significantly to Joule heating within our current dataset.

The longest sequence of spectra that we feel confident in using for this study span approximately $250 \mathrm{~s}$. This is a significant fraction of the thermospheric response time of around $1500 \mathrm{~s}$, and it is thus unlikely that fluctuations on a time scale much longer than this will contribute significantly to the enhanced Joule heating considered by Smith et al. (2005) since some thermospheric damping of the effect will occur. Fluctuations occurring over a shorter time scale than we tested might also occur and, since the thermosphere could not respond to these, would be important. What this study has provided is, therefore, a significant upper limit to the timescale for variations in the atmospheric current that can contribute to the "missing" heating in the upper atmosphere via the Smith et al. (2005) mechanism of enhanced Joule heating.

One explanation as to why we did not observe fluctuations on the timescale of one minute or so, even though Saur et al. (2002) did see them in the magnetosphere, could be that the
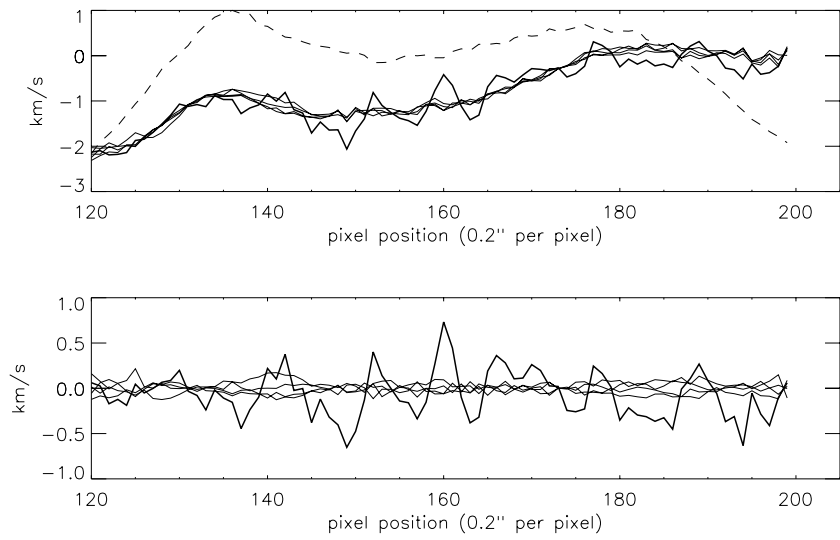

Fig. 5. The four spectra from Group 2 are shown (top) with variations from the group average (bottom). The bold lines are the model profile and fluctuations from the Group 2 average assuming $s=0.5$, smoothed to three pixels to estimate seeing effects. As in Fig. 4, the large negative velocities seen between pixels $\sim 122$ and 132 are due to uneven illumination across the slit as it crosses the dawn limb of the planet. Note that the velocity structure is the same as in Fig. 4. The dashed curve is the average intensity profile for this group of spectra, scaled to the velocity.

Alfvén travel time, around $7500 \mathrm{~s}$, is sufficiently long that these fluctuations are damped out en route from the middle magnetosphere to the upper atmosphere. For our observational configuration, CSHELL's effective spatial resolution is about $640 \mathrm{~km}$ by $640 \mathrm{~km}$ and it is also possible that the fluctuations that contribute significantly to Joule heating are on a smaller spatial scale, such that we miss them in our observations; successful observations of electric field fluctuations at Earth used spatial scales far finer than ours (Aruliah et al., 2005).

Codrescu et al. (1995) pointed out that the Joule heating from fluctuations will dominate in regions where the average electric field is small. For Jupiter, this does not correspond to main auroral oval, but to the dark polar region where the solar wind exerts control over emissions via the Dungey cycle (Dungey, 1961) and the aurora is dimmer and more diffuse. The bright and continuous emissions in the main auroral ovals are driven by the much stronger mechanism of the breakdown of the plasma sheet's corotation with the planet. Within the polar region connected to the open field lines, the emissions are weaker and have been shown to vary considerably (Grodent et al., 2003). Short timescale observations of this region may be more successful in observing fluctuations. Weak mid- to low-latitude emissions have also been detected by Miller et al. (1997). In these regions, away from the main oval, Joule heating from current fluctuations may also be important. 


\section{Conclusions}

Our results are from the first attempt to probe the idea of Joule heating from short-timescale electric field fluctuations in the Jovian upper atmosphere using observational techniques. We have shown that on the timescale of one minute there is no variability in the Jovian auroral ion velocities, averaged over spatial scales of $640 \mathrm{~km}$. This provides an upper limit on the timescale of fluctuations that would contribute to enhanced Joule heating. Future observations, using shorter integration times, will be able to examine shorter timescale variations. They will perhaps also need to coincide with those occasions when clear views of the polar cap regions are available.

Acknowledgements. It is a pleasure to acknowledge the IRTF staff for their expert assistance during our observations. T. Stallard was supported by a UK Particle Physics and Astronomy Research Council (PPARC) postdoctoral fellowship. C. G. A. Smith was funded by a Case studentship from Sun Microsystems and PPARC. The team reporting here is part of the EuroPlaNet European planetary science network, which is supported by the European Union Framework 6 programme. We express our thanks to the anonymous referee who contributed helpful comments that improved this paper.

Topical Editor M. Pinnock thanks two referees for their help in evaluating this paper.

\section{References}

Aruliah, A. L., Griffin, E. M., Aylward, A. D., Ford, E. A. K., Kosch, M. J., Davis, C. J., Howells, V. S. C., Pryse, S. E., Middleton, H. R., and Jussila, J.: First direct evidence of meso-scale variability on ion-neutral dynamics using co-located tristatic FPIs and EISCAT radar in Northern Scandinavia, Ann. Geophys., 23, 147-162, 2005,

http://www.ann-geophys.net/23/147/2005/.

Atreya, S. K.: Atmospheres and Ionospheres of the Outer Planets and their Satellites, Atmospheres and Ionospheres of the Outer Planets and their Satellites, XIII, 224 pp. 90 figs. (partly in color), Springer-Verlag Berlin Heidelberg New York, also: Phys. Chem. Space, volume 15, 1986.

Codrescu, M. V., Fuller-Rowell, T. J., and Foster, J. C.: On the importance of E-field variability for Joule heating in the highlatitude thermosphere, Geophys. Res. Lett., 22, 2393-2396, doi:10.1029/95GL01909, 1995.

Connerney, J. E. P., Acuña, M. H., Ness, N. F., and Satoh, T.: New models of Jupiter's magnetic field constrained by the Io flux tube footprint, J. Geophys Res., 103, 11929-11940, doi:10.1029/97JA03726, 1998.

Cowley, S. W. H. and Bunce, E. J.: Origin of the main auroral oval in Jupiter's coupled magnetosphere-ionosphere system, Planet. Space Sci., 49, 1067-1088, 2001.

Cowley, S. W. H., Bunce, E. J., and O'Rourke, J. M.: A simple quantitative model of plasma flows and currents in Saturn's polar ionosphere, J. Geophys. Res. (Space Physics), 109, 5212, doi:10.1029/2003JA010375, 2004

Cowley, S. W. H., Alexeev, I. I., Belenkaya, E. S., Bunce, E. J., Cottis, C. E., Kalegaev, V. V., Nichols, J. D., Prangé,
R., and Wilson, F. J.: A simple axisymmetric model of magnetosphere-ionosphere coupling currents in Jupiter's polar ionosphere, J. Geophys. Res. (Space Physics), 110, 11209 , doi:10.1029/2005JA011237, 2005.

Dungey, J. W.: Interplanetary Magnetic Field and the Auroral Zones, Phys. Rev. Lett., 6, 47-48, doi:10.1103/PhysRevLett.6.47, 1961.

Greene, T. P., Tokunaga, A. T., Toomey, D. W., and Carr, J. B.: CSHELL: a high spectral resolution 1-5 $\mu \mathrm{m}$ cryogenic echelle spectrograph for the IRTF, in: Proc. SPIE Vol. 1946, p. 313-324, Infrared Detectors and Instrumentation, edited by: Fowler, A. M., pp. 313-324, 1993.

Grodent, D. and Gérard, J.-C.: A self-consistent model of the Jovian auroral thermal structure, J. Geophys Res., 106, 12 933-12952, doi:10.1029/2000JA900129, 2001.

Grodent, D., Clarke, J. T., Waite, J. H., Cowley, S. W. H., Gérard, J.-C., and Kim, J.: Jupiter's polar auroral emissions, J. Geophys. Res. (Space Physics), 108, 6-1, doi:10.1029/2003JA010017, 2003.

Hickey, M. P., Walterscheid, R. L., and Schubert, G.: Gravity Wave Heating and Cooling in Jupiter's Thermosphere, Icarus, 148, 266-281, doi:10.1006/icar.2000.6472, 2000.

Hill, T. W.: Inertial limit on corotation, J. Geophys Res., 84, 6554 6558, 1979.

Matcheva, K. I. and Strobel, D. F.: Heating of Jupiter's Thermosphere by Dissipation of Gravity Waves Due to Molecular Viscosity and Heat Conduction, Icarus, 140, 328-340, doi:10.1006/icar.1999.6151, 1999.

Melin, H., Miller, S., Stallard, T., and Grodent, D.: Non-LTE effects on $\mathrm{H}_{3}^{+}$emission in the jovian upper atmosphere, Icarus, 178, 97 103, doi:10.1016/j.icarus.2005.04.016, 2005.

Melin, H., Miller, S., Stallard, T., Smith, C., and Grodent, D.: Estimated energy balance in the jovian upper atmosphere during an auroral heating event, Icarus, 181, 256-265, doi:10.1016/j.icarus.2005.11.004, 2006.

Miller, S., Achilleos, N., Ballester, G. E., Lam, H. A., Tennyson, J., Geballe, T. R., and Trafton, L. M.: Mid-toLow Latitude $\mathrm{H}_{3}^{+}$Emission from Jupiter, Icarus, 130, 57-67, doi:10.1006/icar.1997.5813, 1997.

Miller, S., Achilleos, N., Ballester, G. E., Geballe, T. R., Joseph, R. D., Prangé, R., Rego, D., Stallard, T., Tennyson, J., Trafton, L. M., and Waite, Jr., J. H.: The role of $\mathrm{H}_{3}^{+}$in planetary atmospheres, in: Astronomy, physics and chemistry of $\mathrm{H}_{3}^{+}$, pp. 2485-2502, 2000.

Millward, G., Miller, S., Stallard, T., Achilleos, N., and Aylward, A. D.: On the dynamics of the jovian ionosphere and thermosphere., Icarus, 173, 200-211, doi:10.1016/j.icarus.2004.07.027, 2005.

Rego, D., Achilleos, N., Stallard, T., Miller, S., Prangé, R., Dougherty, M., and Joseph, R. D.: Supersonic winds in Jupiter's aurorae, Nature, 399, 121, doi:10.1038/20121, 1999.

Saur, J., Politano, H., Pouquet, A., and Matthaeus, W. H.: Evidence for weak MHD turbulence in the middle magnetosphere of Jupiter, Astronomy \& Astrophysics, 386, 699-708, doi:10.1051/0004-6361:20020305, 2002.

Schubert, G., Hickey, M. P., and Walterscheid, R. L.: Heating of Jupiter's thermosphere by the dissipation of upward propagating acoustic waves, Icarus, 163, 398-413, doi:10.1016/S0019-1035(03)00078-2, 2003. 
Seiff, A., Kirk, D. B., Knight, T. C. D., Young, L. A., Milos, F. S., Venkatapathy, E., Mihalov, J. D., Blanchard, R. C., Young, R. E., and Schubert, G.: Thermal structure of Jupiter's upper atmosphere derived from the Galileo probe, Science, 276, 102-104, 1997.

Smith, C. G., Aylward, A. D., and Miller, S.: Magnetospheric Energy Inputs Into the Thermospheres of Jupiter and Saturn, AGU Spring Meeting Abstracts, pp. A1+, 2005.

Southwood, D. J. and Kivelson, M. G.: A new perspective concerning the influence of the solar wind on the Jovian magnetosphere, J. Geophys Res., 106, 6123-6130, doi:10.1029/2000JA000236, 2001.

Stallard, T., Miller, S., Millward, G., and Joseph, R. D.: On the Dynamics of the Jovian Ionosphere and Thermosphere. I. The Measurement of Ion Winds, Icarus, 154, 475-491, doi:10.1006/icar.2001.6681, 2001.
Stallard, T., Miller, S., Millward, G., and Joseph, R. D.: On the Dynamics of the Jovian Ionosphere and Thermosphere. II. The Measurement of $\mathrm{H}_{3}^{+}$Vibrational Temperature, Column Density, and Total Emission, Icarus, 156, 498-514, doi:10.1006/icar.2001.6793, 2002.

Stallard, T. S., Miller, S., Trafton, L. M., Geballe, T. R., and Joseph, R. D.: Ion winds in Saturn's southern auroral/polar region, Icarus, 167，204-211, doi:10.1016/S0019-1035(03)00285-9, 2004.

Strobel, D. F. and Smith, G. R.: On the Temperature of the Jovian Thermosphere., J. Atmos. Sci., 30, 718-725, 1973.

Waite, J. H., Cravens, T. E., Kozyra, J., Nagy, A. F., Atreya, S. K., and Chen, R. H.: Electron precipitation and related aeronomy of the Jovian thermosphere and ionosphere, J. Geophys Res., 88, 6143-6163, 1983.

Young, L. A., Yelle, R. V., Young, R., Seiff, A., and Kirk, D. B.: Gravity Waves in Jupiter's Thermosphere, Science, 267, 108111, 1997. 